\title{
Stability limits for the supercooled liquid and superheated crystal of Lennard-Jones particles
}

Ernesto S. Loscar, Daniel A. Martin, and Tomás S. Grigera

Citation: J. Chem. Phys. 147, 034504 (2017); doi: 10.1063/1.4994049

View online: https://doi.org/10.1063/1.4994049

View Table of Contents: http://aip.scitation.org/toc/jcp/147/3

Published by the American Institute of Physics

\section{Articles you may be interested in}

Common behaviors associated with the glass transitions of water-like models

The Journal of Chemical Physics 147, 034505 (2017); 10.1063/1.4993445

Relaxation dynamics and transformation kinetics of deeply supercooled water: Temperature, pressure, doping, and proton/deuteron isotope effects

The Journal of Chemical Physics 147, 034506 (2017); 10.1063/1.4993790

The constant force continuous molecular dynamics for potentials with multiple discontinuities

The Journal of Chemical Physics 147, 034502 (2017); 10.1063/1.4993436

Perspective: Surface freezing in water: A nexus of experiments and simulations

The Journal of Chemical Physics 147, 060901 (2017); 10.1063/1.4985879

Premelting, solid-fluid equilibria, and thermodynamic properties in the high density region based on the Lennard-Jones potential

The Journal of Chemical Physics 147, 144502 (2017); 10.1063/1.4990667

Molecular simulation of caloric properties of fluids modelled by force fields with intramolecular contributions: Application to heat capacities

The Journal of Chemical Physics 147, 034508 (2017); 10.1063/1.4993572

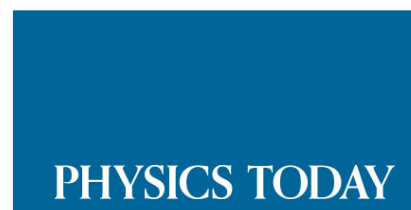

WHITEPAPERS
ADVANCED LIGHT CURE ADHESIVES

Take a closer look at what these environmentally friendly adhesive systems can do

\section{READ NOW}

PRESENTED BY

Q MASTERBOND: 


\title{
Stability limits for the supercooled liquid and superheated crystal of Lennard-Jones particles
}

\author{
Ernesto S. Loscar, ${ }^{1,2,3}$ Daniel A. Martin, ${ }^{4}$ and Tomás S. Grigera ${ }^{1,2,3, a)}$ \\ ${ }^{1}$ Instituto de Física de Líquidos y Sistemas Biológicos (IFLYSIB), CONICET and Facultad de Ciencias Exactas, \\ Universidad Nacional de La Plata, Calle 59 No. 789, B1900BTE La Plata, Argentina \\ ${ }^{2}$ CCT CONICET La Plata, Consejo Nacional de Investigaciones Científicas y Técnicas, La Plata, Argentina \\ ${ }^{3}$ Departamento de Física, Universidad Nacional de La Plata, C.C. 67, 1900 La Plata, Argentina \\ ${ }^{4}$ Instituto de Investigaciones Físicas de Mar del Plata (IFIMAR), CONICET and Universidad Nacional de Mar \\ del Plata, Funes No. 3350, 7600 Mar del Plata, Argentina
}

(Received 21 April 2017; accepted 1 July 2017; published online 18 July 2017)

\begin{abstract}
We have studied the limits of stability in the first order liquid-solid phase transition in a LennardJones system by means of the short-time relaxation method and using the bond-orientational order parameter $Q_{6}$. These limits are compared with the melting line. We have paid special attention to the supercooled liquid, comparing our results with the point where the free energy cost of forming a nucleating droplet goes to zero. We also indirectly estimate the dimension associated to the critical nucleus at the spinodal, expected to be fractal according to mean field theories of nucleation. Published by AIP Publishing. [http://dx.doi.org/10.1063/1.4994049]
\end{abstract}

\section{INTRODUCTION}

The liquid-solid transition is first-order and is thus accompanied by hysteresis: when heating, melting is not observed at the coexistence temperature $T_{m}$ (though it is called melting temperature) but at some higher temperature (conversely when cooling, crystallization occurs at some lower temperature). ${ }^{1-3}$ The liquid phase is metastable below $T_{m}$, which means that although the thermodynamically stable (lowest free energy) phase is the solid, the liquid continues to be locally stable. The metastable phase becomes unstable sufficiently far from the coexistence temperature. In mean-field, the instability appears at a well-defined point, the thermodynamic spinodal, which strictly corresponds to a point where susceptibilities like isothermal compressibility and isobaric heat capacity diverge. This is well defined because in the mean field, the lifetime of the metastable phase is infinite and (meta-)equilibrium measurements are possible.

Outside the mean field, the metastable phase has a finite lifetime, and this time can become of the same order of the relaxation time, thus establishing an effective limit for the existence of the phase (called kinetic spinodal or metastability limit) at a point where the susceptibilities are growing but finite. ${ }^{4-9}$ Extrapolating the susceptibilities beyond the metastability limit, one can define the analogue of the thermodynamic spinodal as the point where the extrapolated susceptibilities seem to diverge. This point is sometimes called pseudospinodal, to emphasize its definition through extrapolation to an unobservable state.

The mean-field spinodal looks like a second-order-phasetransition critical point in that the development of an instability produces a diverging susceptibility (corresponding to a

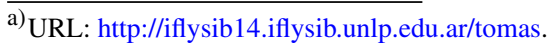

flat free energy). The critical behavior of second-order phase transitions has a particular manifestation in the short-time, outof-equilibrium dynamics, ${ }^{10}$ with several quantities displaying power-law behavior (see Sec. II A). Based on this result, the short-time dynamics (STD) technique ${ }^{11,12}$ has been widely used to determine critical points with out-of-equilibrium measurements. Interpreting the power-law short-time dynamics characteristic of critical points as a signature of the development of an instability, some of us proposed in Ref. 9 to look for such behavior in the vicinity of a first-order transition, and to define in this way a spinodal in finite dimension. This was shown to be consistent because the spinodal defined through STD was found to coincide with the thermodynamic spinodal in the mean-field Ising model, and with the pseudospinodal obtained from extrapolation of the magnetic susceptibility in the short-range Potts model. ${ }^{9}$ Those first results concerned only lattice models, but more recently, using density as an order parameter, the technique was applied to find the spinodals and critical point of the liquid-gas transition of an off-lattice Lennard-Jones fluid. ${ }^{13}$

In this paper, we determine the (pseudo)spinodals of the liquid-solid transition in a Lennard-Jones (LJ) system, as defined by short-time dynamics. In short-range systems spinodals are hard to determine because they involve large relaxation times competing with a shortening lifetime of the metastable phase. The metastable phase becomes in practice unobservable when its lifetime is very short or when its relaxation time is very large. However, the metastable phase could exist, at least in theory, beyond this practical limit, and knowledge of the actual theoretical feasibility of the metastable phase is important in cases such as the thermodynamic (Kauzmann) glass transition (which would lie in a region where the extremely large relaxation times prevent its observation), ${ }^{14,15}$ and the liquid-liquid phase transition of water (not directly observable because of the "no man's land" 
phenomenon). ${ }^{16-18}$ Knowledge of the spinodal can serve as a bound to the region of existence of the metastable phase. The non-equilibrium nature of the STD technique makes it in principle feasible to apply it to these situations but good understanding of the technique and its limitations is needed. In particular, a good order parameter needs to be found, since STD is based on the evolution of the order parameter and its fluctuations.

The aim of this work is to polish the STD technique as applied to the solid-liquid spinodals, studying a well-known model where results with other methods are available for comparison. Thus apart from the interest of the Lennard-Jones model itself, an important result of this work is a perfected technique suitable for the determination of liquid-solid spinodal that does not need equilibrium measurements. In particular, we have paid attention to finding the ordered parameter that best differentiates between the phases and whose fluctuations can signal the destabilization. ${ }^{19}$ We consider the potential energy and the density (used, e.g., in Refs. 20 and 21) but find the best results are obtained with the bond orientational parameter $Q_{6}$ of Steindhardt et al. ${ }^{22}$

The paper is organized as follows: In Sec. II, we give a theoretical description of STD and show how it can be used for studying spinodals. We also show how to calculate the fractal dimension of a crystal nucleus using critical exponents. In Sec. III, we describe the shifted force Lennard-Jones (sfLJ) model and bond orientational order parameter. In Sec. IV, we show our results for the liquid spinodal. We calculate the liquid spinodal for the sfLJ model and estimate the fractal dimension of the critical solid nucleus. We also compare our results with results from TPS. In Sec. V, we show our results for the solid spinodal. Finally, in Sec. VI, we draw our conclusions.

\section{THEORETICAL BACKGROUND}

\section{A. Short time dynamics and spinodals}

Criticality manifests itself not only in the static behavior, but also in relaxation to equilibrium starting from special macrostates. ${ }^{10,23}$ STD can be applied in order to estimate the locus of spinodals and critical points without necessity to reach the equilibrium. Starting from the well defined disordered (high temperature) initial macrostate, where the order parameter $M_{0} \rightarrow 0$ vanishes, the evolution of the order parameter and its fluctuations fulfills

$$
\begin{gathered}
\langle M(t)\rangle \propto M_{0} t^{\theta}, \\
M^{(2)}(t) \sim t^{\psi}=t^{d / z-2 \beta / z v},
\end{gathered}
$$

where $M$ is the order parameter, $d$ is the Euclidean dimension, $\beta$ and $v$ are the order-parameter and the correlation-length critical exponents, respectively, $z$ is the dynamic exponent, and $\theta$ is a new exponent (the STD exponent). The global order parameter is assumed to be a sum of local variables $s_{i}: M$ $=\frac{1}{N} \sum_{i=1}^{N} s_{i}$. The second moment of $M$, at fixed time $t$, is

$$
M^{(2)}(t)=\left\langle[M(t)-\langle M(t)\rangle]^{2}\right\rangle,
$$

where $\langle\cdot\rangle$ is an ensemble average, which in simulations translates to average, at fixed time $t$, over independent runs (trajectories).
The self-correlation of the local variable $s_{i}$,

$$
A\left(t, t_{0}\right)=\frac{1}{N} \sum_{i=1}^{N}\left(\left\langle s_{i}(t) s_{i}\left(t_{0}\right)\right\rangle-\left\langle s_{i}(t)\right\rangle\left\langle s_{i}\left(t_{0}\right)\right\rangle\right)
$$

also behaves as a power law,

$$
A(t) \propto t^{-\lambda}=t^{-(d / z-\theta)} .
$$

Considering instead an initially ordered macrostate $(M$ $=M_{\max }$, low temperature), at criticality, it will decay as

$$
\langle M(t)\rangle \propto t^{-\beta / v z},
$$

and Eq. (2) is also verified. Relations (1), (2), (5), and (6), were originally obtained and verified at second-order critical points. ${ }^{11,12}$

It was first noted by De Gennes and Prost $^{24}$ (for liquid crystals) and by Fernández et $a l .{ }^{25}$ (for the Potts model) that in the case of weak first-order transitions, there are two temperatures around the transition $T_{m}$, namely, $T^{(-)}<T_{m}<T^{(+)}$, where equilibrium data show pseudo-critical behavior. The existence of these temperatures inspired the idea of applying the STD technique to determine $T^{(-)}$and $T^{(+)}$, defined as those where one obtains a power law when starting from a disordered or ordered initial states, respectively. ${ }^{26}$ So STD was used to distinguish first- from second-order phase transitions, by measuring the difference $T^{(+)}-T^{(-)}$, which vanishes in the second-order case. Building on this idea, and based on the fact that the developing of an instability makes the spinodal similar to a second-order critical point, the temperatures $T^{(+)}$and $T^{(-)}$were interpreted as thermodynamic spinodals and shown to coincide with mean field spinodals ${ }^{9,27}$ or pseudospinodals in lattice ${ }^{9,12}$ and more recently off-lattice ${ }^{13}$ systems. Then, in the specific case of the liquid-solid transition, we identify $T^{(-)}$ with the liquid spinodal $\left(T_{s p}^{* L i q}\right)$ and $T^{(+)}$with the solid spinodal $\left(T_{s p}^{* S o l}\right)$.

\section{B. Spinodal fractal dimension}

At a second-order critical point, the largest clusters belonging to the low temperature phase are fractal-like objects with fractal dimension

$$
d_{f}=d-\beta / v .
$$

Analogously, mean field theories such as the spinodal nucleation theory ${ }^{28,29}$ conclude that nucleating droplets at the spinodal temperature are fractal-like objects with divergent number of particles. Signs of this characteristic behavior have been obtained also in several short range systems. ${ }^{30-32}$

In critical points, it is usually possible to estimate $\beta / v$ by means of STD and, therefore, $d_{f}$ of Eq. (7). It can be done using relations (1), (2), and (5). From Eq. (2), we have $\beta / v$ $=(d-\psi z) / 2$ and using Eq. (5), we have $\beta / v=\frac{d}{2}\left(1-\frac{\psi}{\lambda+\theta}\right)$. Substituting in Eq. (7), we obtain

$$
d_{f}=\frac{d}{2}\left(1+\frac{\psi}{\lambda+\theta}\right)
$$

Analogously, in the case of spinodals, the dimension $d_{f}$ obtained from $M, M^{(2)}$, and $A(t)$ [Eqs. (1), (2), and (5)] would correspond to the fractal dimension of the nucleating droplet. 


\section{SIMULATION DETAILS}

\section{A. Model}

On the one hand, we have used a shifted force LennardJones (sfLJ) system of $N=2048$ particles with the interacting potential

$$
V(r)= \begin{cases}4 \epsilon\left[\left(\frac{\sigma}{r}\right)^{12}-\left(\frac{\sigma}{r}\right)^{6}\right]+A r+B, & r \leq r_{c}, \\ 0, & r>r_{c},\end{cases}
$$

with $A$ and $B$ constants that ensure continuity of $V(r)$ and $V^{\prime}(r)$ at $r=r_{c} .{ }^{33}$ This simple scheme is computationally convenient because no corrections to the energy due to the tail and cutoff are needed. We set $r_{c}=2.5 \sigma$ as in the original paper of Errington et al. ${ }^{33}$ where the phase diagram was determined. This potential is used in Secs. IV A-IV C and V.

On the other hand, in order to compare with Ref. 31, in Sec. IV D, we have used the Lennard-Jones potential argon parameters, namely, $\epsilon / k_{B}=119.8 \mathrm{~K}$ (where $k_{\mathrm{B}}$ is Boltzmann's constant) and $\sigma=3.405 \AA .{ }^{34}$ Also, as in Ref. $31 A=B=0$, $r_{c}=9 \AA$ in Eq. (9), with the usual long range correction for energy and pressure. ${ }^{35}$

We have done Monte Carlo simulations with the Metropolis algorithm in the NPT ensemble with periodic boundary conditions. Two kinds of move attempts are allowed: particle shifts (with probability $p_{s}=0.9995$ ) and volume changes (with probability $\left.1-p_{s}\right)$. Particle shifts consist of random displacements up to a maximum distance $d_{r}=0.075$. Volume changes are limited to a $1 \%$ decrease or increase, keeping a cubic simulation shape. As usual, progress in the simulation is measured in Monte Carlo Steps (MCS), i.e., the number of attempted moves per particle. We express the results in reduced units, $T^{*}=k_{\mathrm{B}} T / \epsilon, p^{*}=p \epsilon / \sigma^{3}, \rho^{*}=\rho \sigma^{3}$, and $u^{*}=u / \epsilon$.

\section{B. Order parameter}

To study crystallization, we use the global orientational parameters $Q_{l}{ }^{22}$ For a given configuration it is defined by

$$
Q_{l}=\sqrt{\frac{4 \pi}{2 l+1} \sum_{m=-l}^{m=l}\left|\left\langle Q_{l m}\left(\hat{\mathbf{r}}_{i j}\right)\right\rangle_{b}\right|^{2}},
$$

where $\hat{\mathbf{r}}_{i j}=\left(\mathbf{r}_{j}-\mathbf{r}_{i}\right) /\left|\mathbf{r}_{j}-\mathbf{r}_{i}\right|$ is the bond-versor between neighbor particles $i$ and $j, Q_{l m}\left(\hat{\mathbf{r}}_{i j}\right)=Y_{l m}\left(\theta\left(\hat{\mathbf{r}}_{i j}\right), \phi\left(\hat{\mathbf{r}}_{i j}\right)\right)$, with $\theta$ and $\phi$ are the spherical angles of $\hat{\mathbf{r}}_{i j}, Y_{l m}$ are the spherical harmonics, and $\langle\ldots\rangle_{b}$ means the average over all bonds of the configuration under study. Particles are considered as neighbors if separated by a distance less than the characteristic length $r_{0}=\left(\rho_{0} / \rho\right)^{1 / 3} d_{1}$, where $\rho=N / V$ and $\rho_{0}$ is the unit density. $d_{1}$ is the nearest-neighbor distance at unit density, calculated from $g(r), d_{1} \simeq 1.4$. Results do not sensitively depend on $d_{1}$ (note that $V$ varies along the simulation).

We also define a corresponding local order parameter $q_{l}(i),{ }^{30}$

$$
q_{l}(i)=\sqrt{\frac{4 \pi}{2 l+1} \sum_{m=-l}^{m=l}\left|q_{l m}(i)\right|^{2}} .
$$

Here $q_{l m}(i)$ is the orientational order parameter for the particle $i$,

$$
q_{l m}(i)=\frac{1}{n_{i}} \sum_{j=1}^{n_{i}} Y_{l m}\left(\theta\left(\hat{\mathbf{r}}_{i j}\right), \phi\left(\hat{\mathbf{r}}_{i j}\right)\right),
$$

where the average is taken over $n_{i}$, the nearest neighbors of particle $i$.

The global parameter $Q_{6}$ is highly sensitive to crystallization in the LJ system. ${ }^{33}$ For completely uncorrelated particles, $Q_{6}=1 / \sqrt{N_{b}}$, where the number of bonds is $N_{b}=z N$ ( $z$ is the mean number of neighbors), while for a perfect FCC crystal $Q_{6}=0.57452$. This quantity is widely used in numerical simulations as an indicator of crystallization, for example, to study the clusters of solid-like particles, ${ }^{30,36,37}$ to define free energy barriers for nucleation, ${ }^{31,38}$ to build a transition path sampling (TPS) scheme, ${ }^{31}$ and to distinguish among different kinds of crystals ${ }^{39}$ and liquids. ${ }^{40}$ A similar quantity, which can in principle be experimentally measured, has recently been proposed. $^{41}$

\section{THE LIQUID SPINODAL}

\section{A. Supercooled phases and hysteresis}

Figures 1 and 2 show stationary results of our simulations using the sfLJ potential in the NPT ensemble with $p^{*}=0.50$. In Fig. 1, we show the potential energy per particle $u^{*}$ and
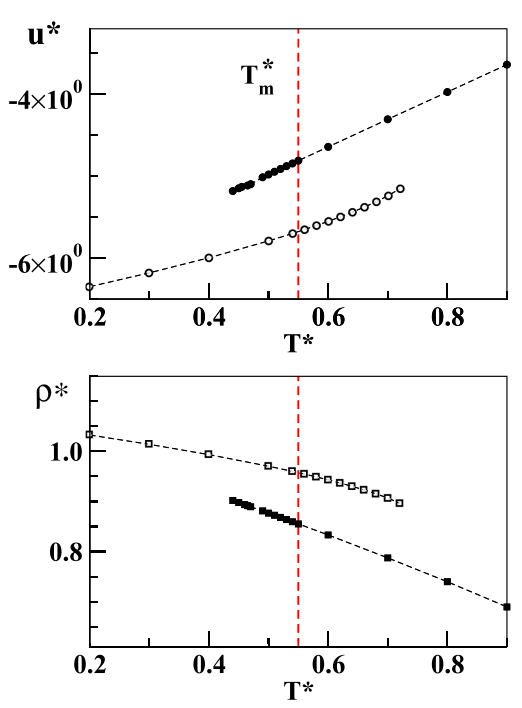
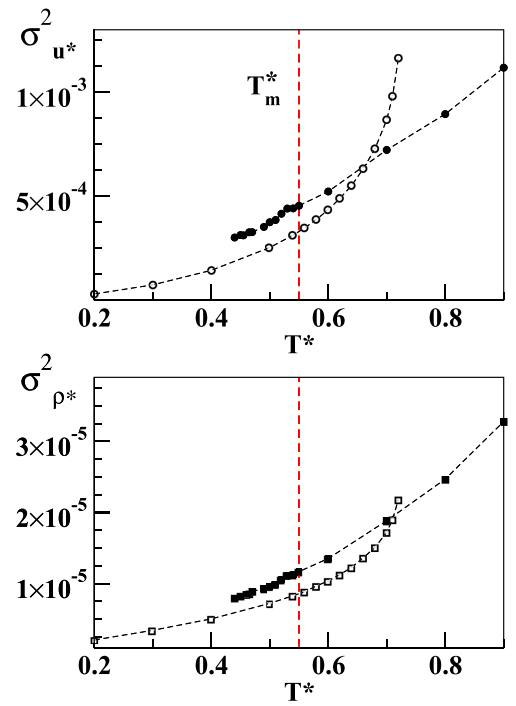

FIG. 1. Stationary results starting from the initial configuration corresponding to a disordered liquid (filled symbols) and a perfect crystal (empty symbols). The left panel shows $u^{*}$ and $\rho^{*}$ obtained using the NTP ensemble with $p^{*}=0.50$ and $N=2048$ for the sfLJ potential given by Eq. (9). Vertical dashed line is the temperature of transitions estimated from Ref. 33. Right panel: Stationary fluctuations of $u^{*}$ and $\rho^{*}$ given by the variance $\sigma_{x}^{2}$. For each temperature, the data were obtained after $5 \times 10^{5}$ MCS, measuring until $t_{o b s}=5 \times 10^{6} \mathrm{MCS}$, and using five independent runs to estimate the statistical error. Symbols are greater than the statistical errors. 

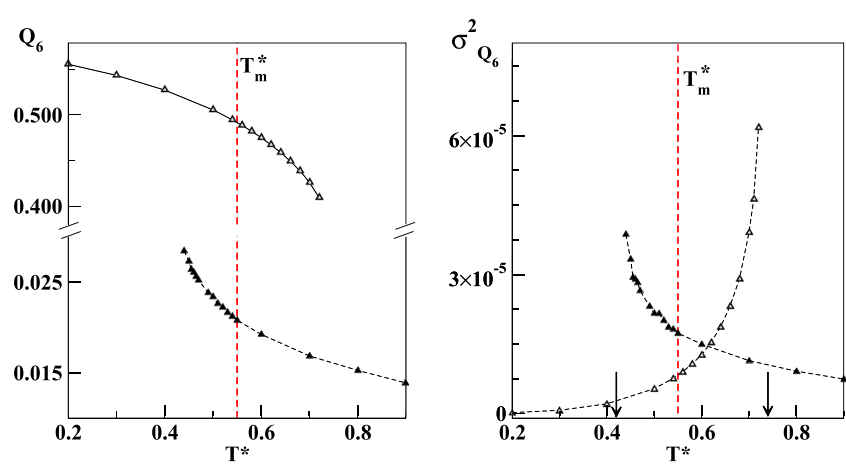

FIG. 2. Idem Fig. 1 but for $Q_{6}$. The arrows indicate the STD spinodal temperatures.

the density $\rho^{*}=N / V^{*}$ together with their fluctuations, given by the variance $\sigma_{x}^{2}=\left\langle x^{2}\right\rangle_{t}-\langle x\rangle_{t}^{2}$, estimated using time averages. In Fig. 2, we show the orientational parameter $Q_{6}$ and its fluctuations. For each temperature, these time averages were obtained after a relaxation stage of $5 \times 10^{5} \mathrm{MCS}$ and then measuring the observables every $100 \mathrm{MC}$ steps up to $t_{o b s}=5 \times 10^{6}$ MCS. Statistical errors were estimated using five independent runs. The melting transition temperature has been calculated in Ref. 33. From this reference, at this pressure, it can be estimated as $T_{m}^{*} \approx 0.55$, which corresponds to the vertical line as indicated in the figures.

The results reported in the figures were obtained starting from two different initial conditions: random positions (infinite temperature) and from a perfect FCC crystal. It is clear that the different initial conditions result in different values of the observables, and that no discontinuity is observed as the temperature is varied across $T_{m}^{*}$. The figures clearly show that both the liquid and solid phases can become metastable.

The data for disordered initial configurations in Figs. 1 and 2 were obtained at temperatures for which the system does not crystallize during the observation time $t_{o b s}=5 \times 10^{6} \mathrm{MCS}$ and has a stationary value of $Q_{6}$ for all the samples. For $T^{*}<0.44$ some signals of crystallization were detected, and therefore we have discarded these temperatures.

Another fact that emerges from the figures is that $Q_{6}$ and its fluctuations show, with respect to density and energy, steeper variations as the temperature moves deeper within the metastable region. This is related to our finding that the orientational parameter is the one suitable to study STD, rather than energy or density, as we discuss in Sec. IV B.

\section{B. The liquid spinodal in the sfLJ potential}

The increase in the fluctuations of $Q_{6}$ in Fig. 2 suggests an instability near $T^{*} \sim 0.44$ (for $p^{*}=0.50$ ). We thus explore temperatures around this point using STD. The initial disordered macrostate required to apply STD in this case is obtained taking the system at infinite temperature, which makes $Q_{6}=O\left(N^{-1 / 2}\right) \simeq 0$. To determine completely the initial macrostate, it is also necessary to select a density. Following Ref. 13, we have chosen an estimate for the spinodal density, which is $\rho_{s p}^{*} \approx 0.9225$. It was obtained, for $p^{*}=0.50$, using the data of Fig. 1 . We have also used different initial densities (within a 10\% range of this value) and checked that our results are independent of the initial density.

We follow the relaxation from the disordered state in the $N P T$ ensemble (Fig. 3). The critical STD behavior is given by the order parameter in Eq. (1) $\langle M(t)\rangle$ (hereafter denoted $M$ for simplicity) and its second moment (i.e., fluctuations) calculated as in Eq. (3). We consider the three quantities $u^{*}, \rho^{*}$, and $Q_{6}$. Density and energy show no sign of the initial increase expected for the order parameter in STD and neither do their fluctuations. It is clear that these quantities are unsuitable to detect the spinodal singularity. Instead, both $Q_{6}$ and its second moment have a clear initial increase, as expected for the order parameter near a singularity. We thus proceed to study the spinodal applying the STD technique to $Q_{6}$.
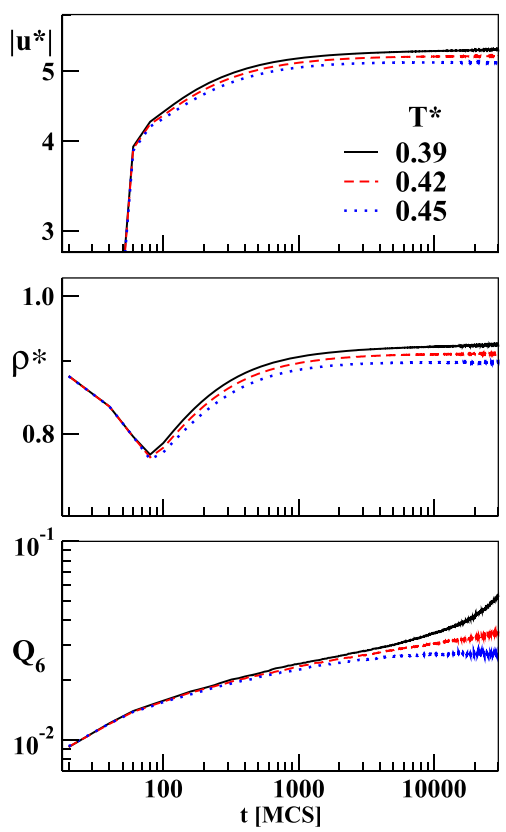
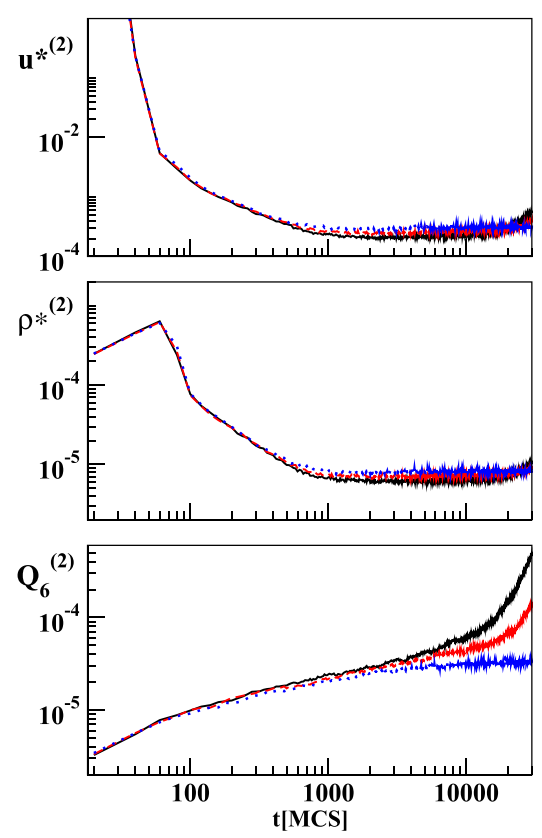

FIG. 3. Results for a system prepared at $T^{*}=\infty$, relaxing at $p^{*}=0.5$, and different temperatures as indicated in the top left panel. The absolute energy $\left|u^{*}\right|$, density $\rho^{*}$, and the parameter $Q_{6}$ are showed on the left panel and its fluctuations on the right panel [second moment calculated with Eq. (3)]. These results correspond to average taken over $10^{3}$ samples. 

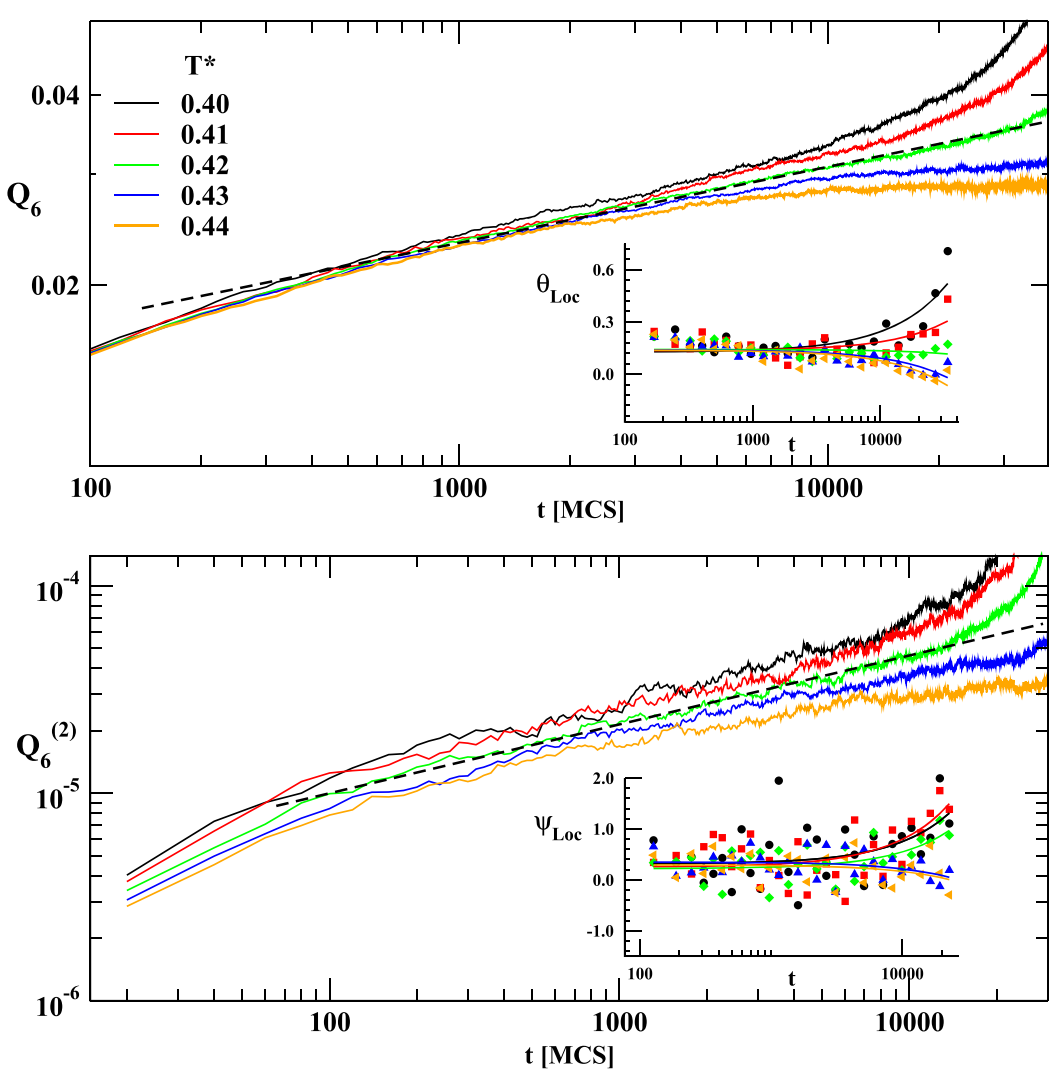

FIG. 4. Results for $Q_{6}$ and its second moment $Q_{6}^{(2)}$ at $p^{*}=0.50$, for a system prepared at $T^{*}=\infty$ and let relax at different temperatures (data for $Q_{6}^{(2)}$ were shifted vertically, except at $T=0.42$, for the sake of clarity). Temperatures are indicated in correspondence with the order in the graph from top to bottom. The insets show, in a log-linear plot, the local exponents $\theta_{L o c}$ and $\psi_{L o c}$ as a function of $t$, while lines are linear fits.
Figures 4 and 5 show the evolution of $Q_{6}$ and its second moment $Q_{6}^{(2)}$ for a range of pressures $\left(p^{*}=0.50,5.00\right.$, and 30 ) and temperatures (as indicated). These results correspond to our STD simulations with $n_{s}=500-1000$ independent samples. We observe for each pressure the initial increase in $Q_{6}$ and in its second moment $Q_{6}^{(2)}$.

In order to find the pseudo-critical temperature, we have computed the local (in time) exponents [analogous to the exponents defined by Eqs. (1) and (2)] $\theta_{L o c}$ and $\psi_{L o c}$ by binning the data in logarithmic time windows and then fitting power laws over consecutive groups of three points. Figure 4 shows $Q_{6}$ and its second moment $Q_{6}^{(2)}$ for $p^{*}=0.50$, while the insets show the corresponding local exponents. In these insets, the solid lines are linear fits that indicate the average trend. We can see that the local exponent $\theta_{\text {Loc }}$ for $T^{*}=0.42$ is the most stable. In the case of the $\psi_{L o c}$, the stable exponent should be in the range $0.42<T^{*}<0.43$. This difference is due to the finite size of the system and tends to disappear for greater sizes. We associate the spinodal temperatures with the (time-limited) power law behavior observed in Figs. 4 and 5. Assuming a power law behavior at $T_{s p}^{* L i q}=0.42$, Fig. 4 shows a fit for $Q_{6}$ valid from $t_{\text {mic }} \sim 500$ to $t_{\text {max }} \sim 32000$ and for $Q_{6}^{(2)}$ from $t_{\text {mic }} \sim 70$ to $t_{\text {max }} \sim 13000$. We note that this value of spinodal is
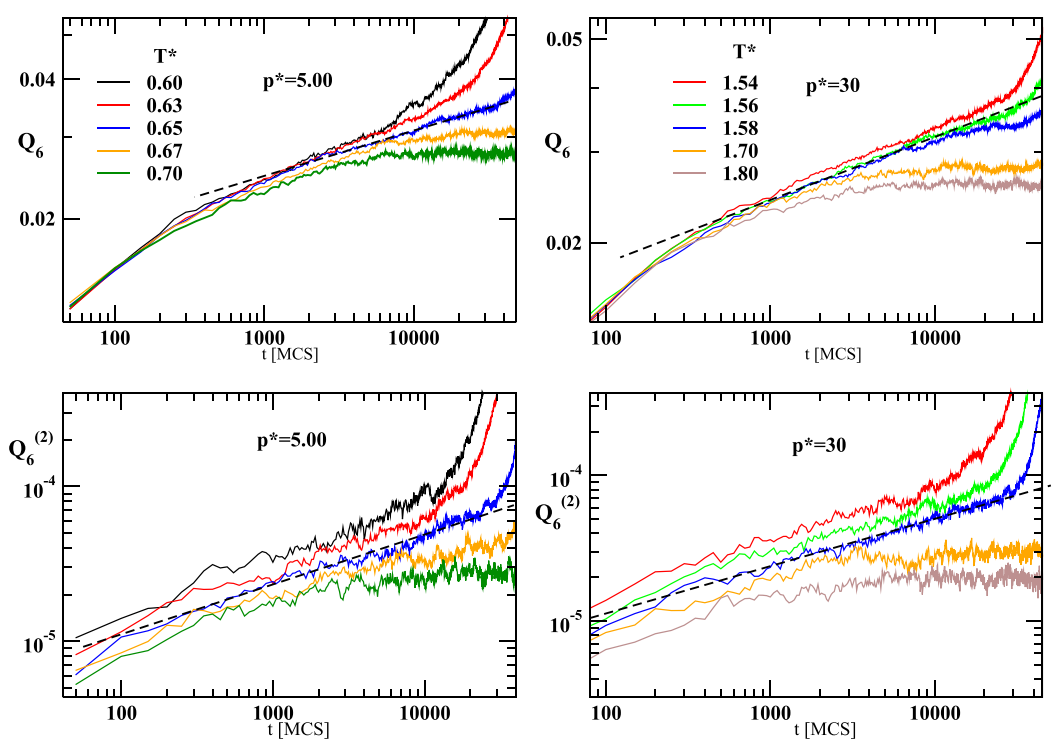

FIG. 5. As in Fig. 4 but for $p^{*}=5.00$ (left) and $p^{*}=30.00$ (right). 


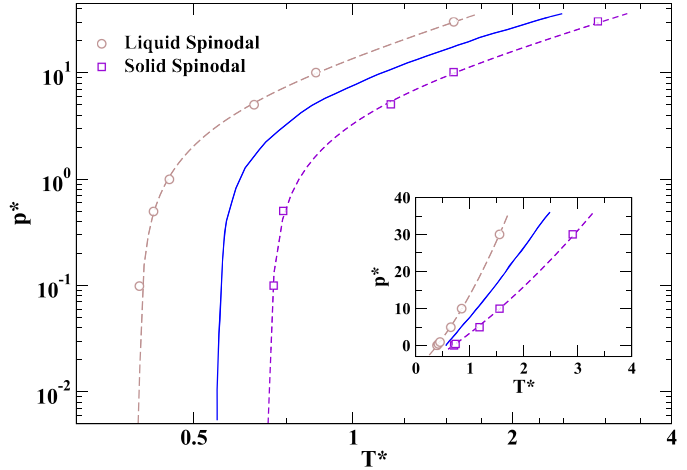

FIG. 6. Phase diagram for the melting transition for LJ system with sfLJ potential. Continuous line shows data of Ref. 33 . The inset shows a linearlinear graph.

compatible with the stationary values shown in Fig. 2 (in the right panel of this figure the left arrow indicates $T_{s p}^{* L i q}$ ).

We have repeated the analysis for different pressures in the range $p^{*}=0.1$ to $p^{*}=30$ and we have found a pseudocritical behavior in all cases for both $Q_{6}$ and $Q_{6}^{(2)}$. In Fig. 6, we plot the spinodal lines determined from $Q_{6}^{(2)}$ together with the coexistence curve taken from Ref. 33. In the linear scale graph (inset), we can observe that the width of the metastable region increases for higher pressure and temperature: the liquid can be supercooled more deeply at high pressures.

\section{Fractal dimension of the critical droplets}

According to the theory of thermal critical phenomena, in critical conditions, the largest condensed cluster is a fractal object. Fractal objects have been reported when the structure of the nucleating droplet near the spinodal is studied. ${ }^{30,31} \mathrm{In}$

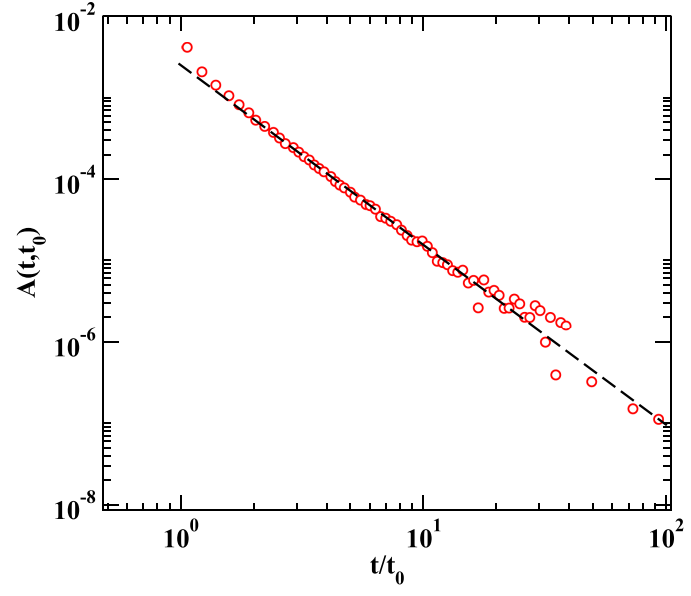

FIG. 7. Results for the autocorrelation of $Q_{6}$ using Eq. (4) for a system prepared at $T^{*}=\infty$ and $t_{0}=120$.

TABLE I. Summary of spinodal exponents and spinodal temperature obtained by applying the STD method. The exponent $\theta$ is obtained with the initial increase of $Q_{6}, \phi$ is obtained with fluctuations, and $\lambda$ with correlation $A(t)$.

\begin{tabular}{lccccc}
\hline \hline$p^{*}$ & $T_{s p}^{* L i q}$ & $\theta$ & $\psi$ & $\lambda$ & $d_{f}=\frac{d}{2}\left(1+\frac{\psi}{\theta+\lambda}\right)$ \\
\hline 0.50 & $0.42(1)$ & $0.13(1)$ & $0.33(1)$ & $2.2(1)$ & $1.71(2)$ \\
5.00 & $0.65(2)$ & $0.11(3)$ & $0.34(2)$ & $2.1(1)$ & $1.73(3)$ \\
30.0 & $1.54(2)$ & $0.130(15)$ & $0.35(3)$ & $2.1(1)$ & $1.74(3)$ \\
\hline \hline
\end{tabular}

the same way as in percolation theory, ${ }^{42}$ the fractal dimension $d_{f}$ of this object can be related to the correlation-length and order-parameter critical exponents ( $v$ and $\beta$, respectively)
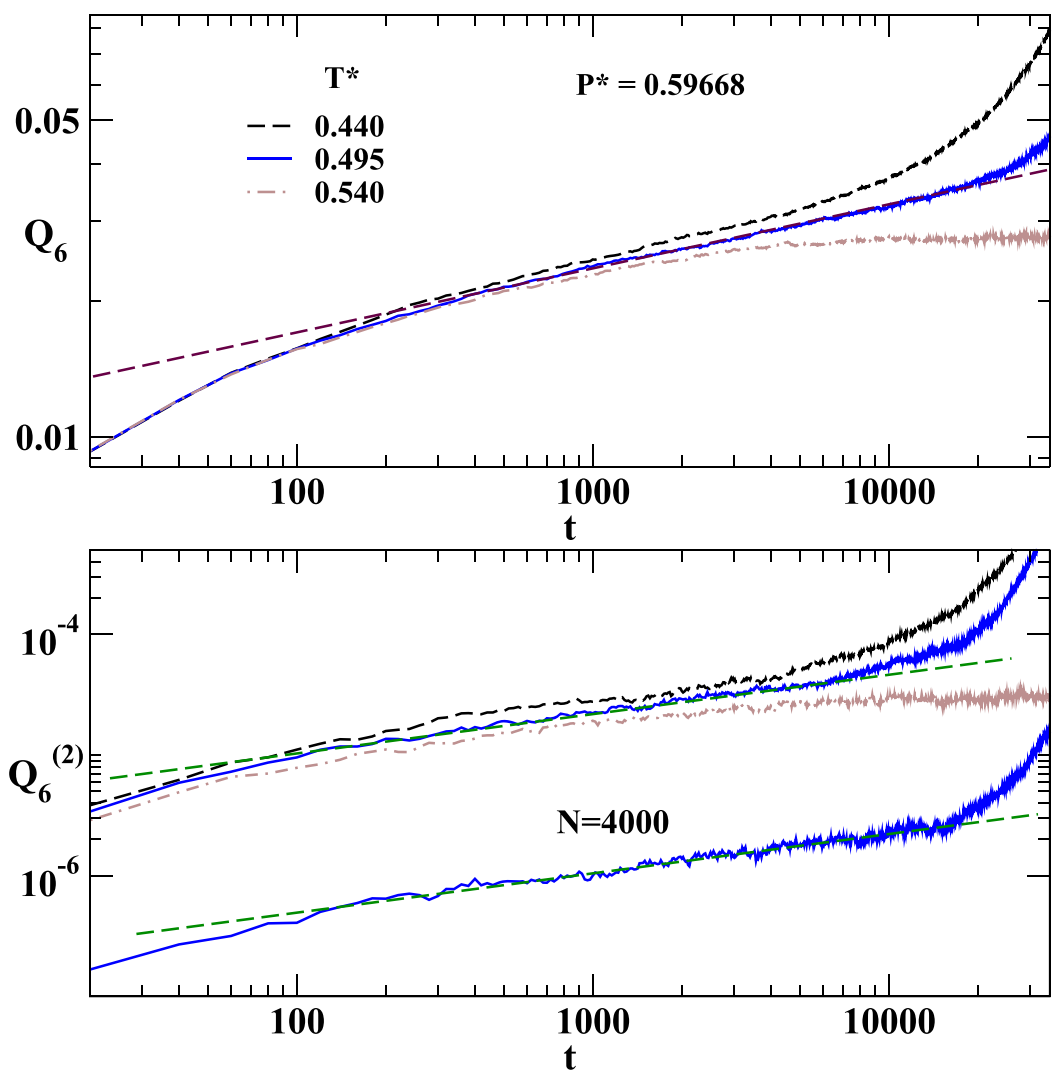

FIG. 8. Liquid spinodal detected by means of STD for the same potential used in Ref. 31 for $p^{*}=0.59668$ (corresponding to $p=250$ bars according to the argon parameters) and obtained for a system of $N=2048$ particles. Top panel: Orientational parameter at indicated temperatures. Bottom panel: Fluctuations of $Q_{6}$ (the data obtained with $N=4000$ are indicated and were shifted down for the sake of clarity). 
through Eq. (7). To determine $d_{f}$, we then need the exponents for the initial increase of the order parameter $\theta$, of its fluctuations $\phi$, and of the self correlation $\lambda$. We have computed the self correlation as given by Eq. (4), using the local orientational parameter for each particle $q_{6}(i)$ [see Eq. (11)].

Figure 7 shows the autocorrelation for $p^{*}=0.50$ and spinodal temperature $T_{s p}^{* L i q}=0.42$ (estimated from Figs. 4 and 5). We remark that the results shown in this figure were obtained from the same STD experiment, i.e., a relaxation from the completely disordered macrostate. The self correlation depends on two times: the time $t_{0}$ when a reference configuration is recorded and the time $t$ elapsed since $t_{0}$. In particular, because this is an out-of-equilibrium process, the correlation is not time-translation invariant and thus the initial time $t_{0}$ is important. To select $t_{0}$, we use the criterion of the best (longer) power law, resulting $t_{0}=120$. Consistently, this time is also $\sim t_{\text {mic }}$, the time since the power law of the order parameter and its moments is valid (see Figs. 4 and 5 for $p^{*}=0.50$ ). From the power law fit of Fig. 7, we obtain $\lambda=2.20$. We have repeated the measurement for different values of $p^{*}$ and we found the same exponent (within the statistical error) for the range of studied pressures. We give in Table I the exponents obtained from Fig. $4(\theta)$ and from Fig. $5(\phi)$ as well as $\lambda$ from the self correlation at different pressures. The last column is the estimate of the fractal dimension $d_{f}$. These exponents are always compatible with the values $\phi=0.34(2), \theta=0.13(1)$, and $\lambda=2.1(2)$, giving $d_{f}=1.73(2)$.

\section{Argon at $\mathbf{2 5 0}$ bars}

We have looked at other estimations of spinodals in the $\mathrm{LJ}$ system in order to compare with our results. To the best of our knowledge, there are no direct determinations of the liquid spinodal against the solid in this system, but in Ref. 31, the limit of the formation free energy of the critical nucleus $\Delta G^{*} \rightarrow 0^{+}$is carefully determined. In principle, this limit is expected to coincide with the thermodynamical spinodal point. ${ }^{43}$

Hence we have also done simulations with the potential used in Ref. 31 for argon at $p=250$ bars. Using the LJ parameters for argon (Sec. III A), we can compute the corresponding reduced pressure as $p^{*}=0.59668$. For this pressure, the melting temperature obtained in Ref. 34 is $T_{m}^{*}=0.75$ \pm 0.01 .
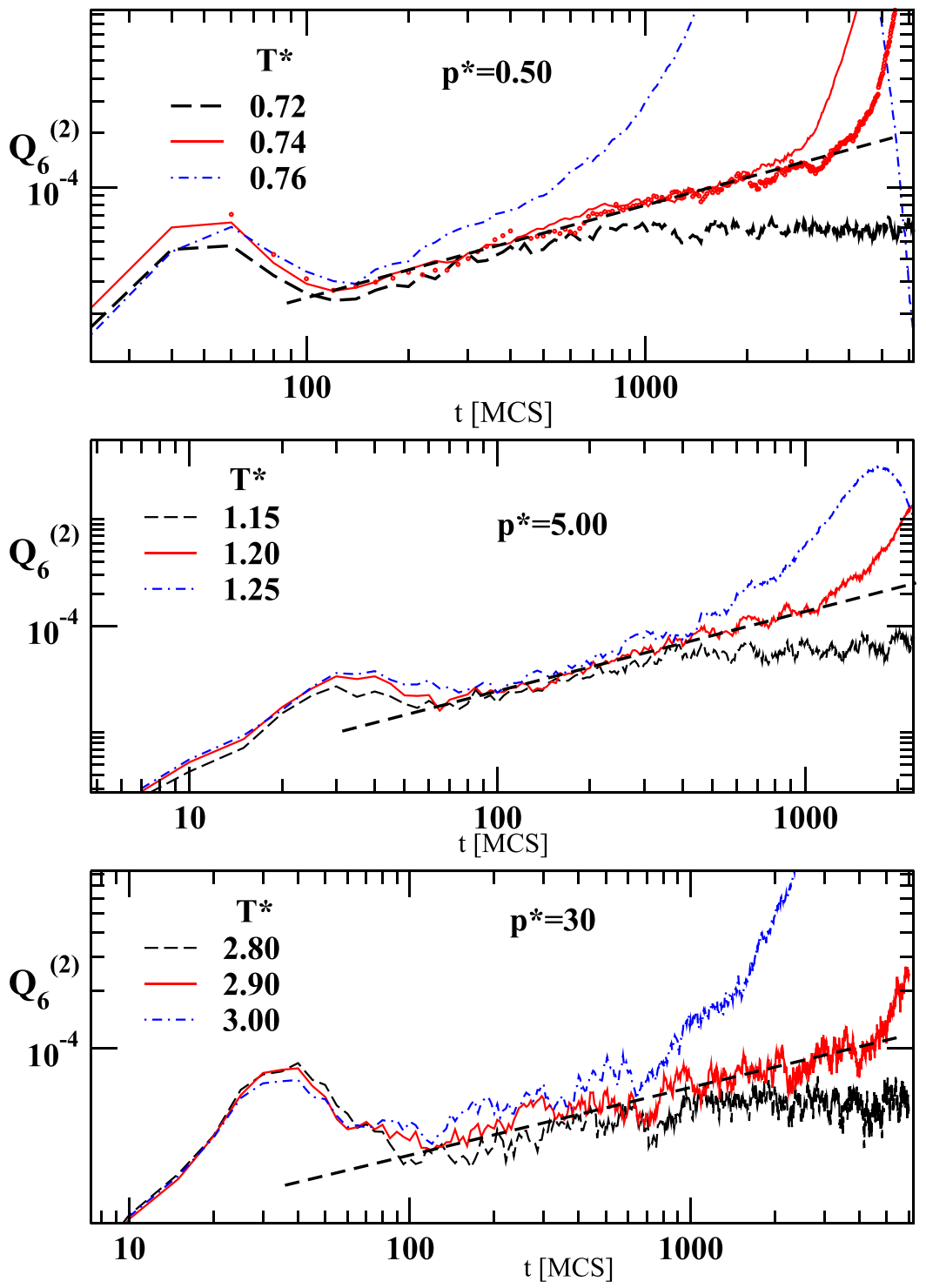

FIG. 9. Results for the second moment $Q_{6}^{(2)}$ of the parameter $Q_{6}$ for a system prepared at $T^{*}=0$ as a FCC lattice and leaving for relaxation at different pressures. Temperatures are indicated in correspondence with the order in the graph from top to bottom. In the top panel, the red points are results obtained for a bigger system of $N=4000$ particles (shifted vertically for sake of clarity). 
Figure 8 shows the results for $Q_{6}$ and $Q_{6}^{(2)}$. Our estimation of the spinodal reduced temperature is $T_{s p}^{* L i q}=0.495 \pm 0.005$, which is equivalent to $T_{s p}^{* L i q} / T_{m}^{*}=0.66 \pm 0.01$. Using a larger size $(N=4000)$, there is no difference in the behavior of $Q_{6}$, but $Q_{6}^{(2)}$ shows a power law over a longer time (Fig. 8).

The critical value of the free energy for the formation of a crystalline nucleus $\Delta G^{*}$ was obtained in Ref. 31, for several temperatures. A linear extrapolation of this value predicts that $\Delta G^{*}$ vanishes at $T^{*} / T_{m}^{*}=0.64$ (a value unfortunately reported without an error). This value is slightly lower than our estimation. However, we note that the measure of $\Delta G^{*}$ were down up to $T^{*} / T_{m}^{*}=0.7$ and a linear extrapolation might not be valid near the spinodal.

\section{THE SOLID SPINODAL}

From Fig. 2, one guesses that $Q_{6}$ is also sensitive to solid spinodal, because its fluctuations increase when the spinodal region is approached, while energy and density are less sensitive. Accordingly, we have attempted to use STD starting with ordered initial conditions. This requires preparing a state with the order parameter at the maximum value (corresponding to zero temperature). We have prepared the system in a perfect FCC crystal, with $Q_{6} \approx 0.5745$. The volume is chosen at a value corresponding to the limit $T^{*} \rightarrow 0^{+}$, for $N=2048$ and $p^{*}=0.50$ of our simulation. It implies an initial density $\rho_{0}^{*} \simeq 1.053$. For simplicity, the same macrostate is used for different pressures.

Figures 9 and 10 show the results of our STD simulations with $n_{s}=100$ independent samples. In Fig. 9, we can observe the second moment $Q_{6}^{(2)}$ for different temperatures. We can clearly identify a characteristic temperature where a power law is observed. For each pressure, this temperature is identified as the solid spinodal point.

Figure 1 suggests that the potential energy $u^{*}$ could be sensitive to the solid spinodal instability. To test this, we plot in Fig. 10 the behavior of the second moment $u^{*(2)}$ for the same pressures studied in Fig. 9. For low pressure $\left(p^{*}=0.50\right)$, we found again a power law behavior at $T^{*}=T_{s p}^{* S o l}=0.74$ confirming the value obtained with $Q_{6}$. Increasing the pressure, at $p^{*}=5.00$, we observe a weak and noisy increment in $u^{*(2)}$, which becomes practically unobservable for a higher pressure $\left(p^{*}=30\right)$. It should be noted that the energy fluctuations are enhanced by increasing the pressure, which makes it more difficult to observe the expected initial increase in $u^{*(2)}$. The behavior of the density (not shown) is qualitatively the same as the potential energy.

Figures 9 and 10 reveal that, although $u^{*}$ can perhaps be used to detect the spinodal instability, $Q_{6}$ is a better choice, which is also in agreement with the stationary results of metastability in Fig. 2.

In Fig. 6, we have plotted the solid spinodal obtained for different pressures together with the coexistence line ${ }^{33}$ (continuous blue line) and the liquid spinodals points. This figure summarizes our STD results, obtained in Secs. IV B and $\mathrm{V}$, using the bond orientational parameter $Q_{6}$ for the sfLJ potential.
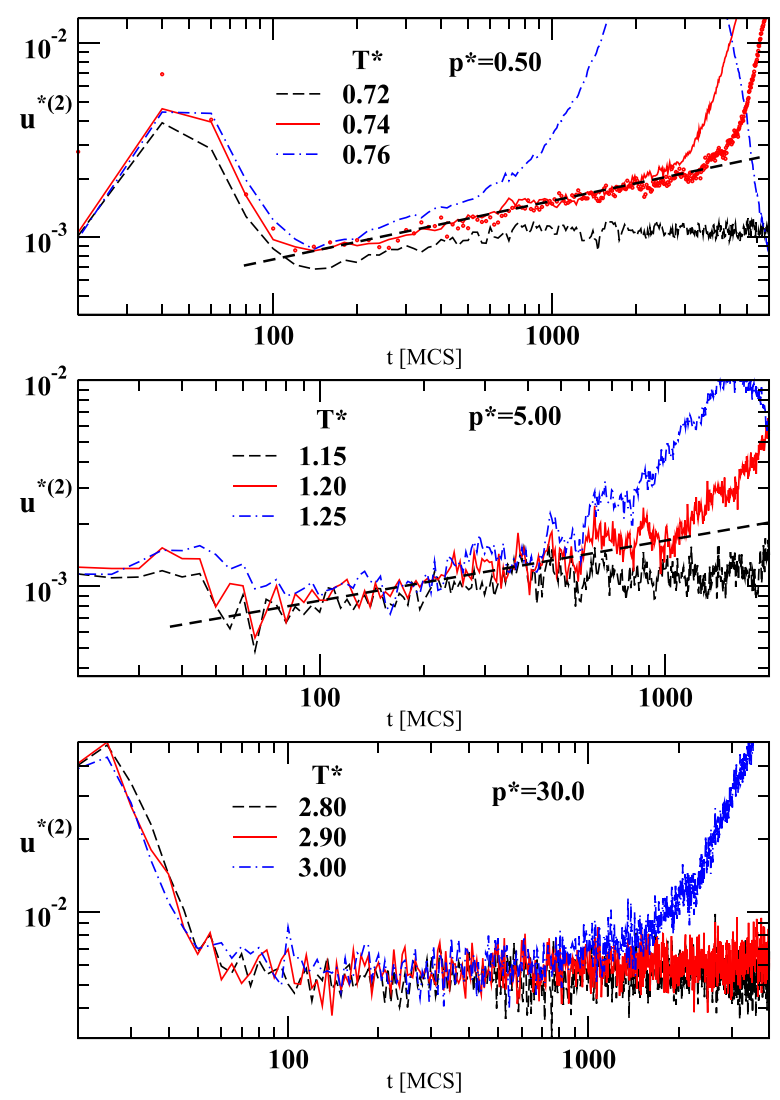

FIG. 10. Results for the second moment of the potential energy for a system prepared at $T^{*}=0$ in a FCC lattice and leaving for relaxation at the indicated pressures and temperatures. Temperatures are indicated in correspondence with the order in the graph from top to bottom. In the top panel, the red points are results obtained for a bigger system of $N=4000$ particles (shifted vertically for the sake of clarity).

\section{CONCLUSIONS}

We have studied the (pseudo)spinodals of the solid-liquid transition in a simple Lennard-Jones model. We have demonstrated, applying the STD technique, that the bond orientational parameter $Q_{6}$ can be used as an order parameter to detect clear signs of instability in two regions close to this transition. We associate these instabilities with the liquid and solid spinodals. In this way, we found that liquid and solid spinodals can be detected using STD, similarly to simple lattice models. To this end, well defined initial macrostates, corresponding to disorder with $Q_{6} \sim 0$ (infinite temperature) and to order with maximum $Q_{6}$ (zero temperature), have been used.

The most relevant result is for the metastable liquid, in which $Q_{6}$ captures the instability against crystallization. In contrast, other thermodynamic quantities like the energy or the density are not sensitive to the instability and instead show smooth variations with temperature. We have compared our spinodal temperature, computed with argon parameters at $p=250$ bars $\left(T_{\mathrm{sp}}^{*} / T_{m}^{*}=0.66 \pm 0.01\right)$ with the results found through extrapolation of the free energy barrier to the vanish$\operatorname{ing}$ point $\left(T_{s p}^{*} / T_{m}^{*} \approx 0.64^{31}\right)$. We find a slightly greater spinodal temperature. This small difference $\sim 3 \%$ seems to suggest that the instability detected by means of STD and the point defined with the vanishing barrier are the same. We remark that the thermodynamic spinodal is commonly expected to coincide 
with that coming from the vanishing barrier. For the solid spinodal, we found that energy or density can be used as an order parameter, but $Q_{6}$ is still preferable because it is more sensitive, especially at high pressure.

Using the local orientational parameter $q_{6}$, we indirectly found the fractal dimension for solid nuclei at the liquid spinodal, obtaining $d_{f}=1.73(2)$. This value is compatible with the universality class of irreversible cluster-cluster aggregation. ${ }^{44}$ This is a kind of diffusion-limited aggregation (DLA) where the structure results from cluster formation by the homogeneous aggregation of collections of particles. In this model, clusters have a fractal dimension $d_{f}=1.75$ (found by simulation $^{44}$ and experimentally in the kinetic aggregation of uniformly sized aqueous gold colloids ${ }^{45}$ ).

In conclusion, the parameter $Q_{6}$, as used here with the STD technique, allows us to study instabilities associated to the liquid-solid spinodal in a convenient and efficient way. We expect that this technique will prove extremely valuable in situations where equilibration of the metastable phase is impossible (such as in deeply supercooled glass-forming liquids) and in complex situations where $Q_{6}$ still serves as a good order parameter sensitive to phase changes. ${ }^{38,39}$

\section{ACKNOWLEDGMENTS}

This work was supported by CONICET, Universidad Nacional de La Plata, Universidad Nacional de Mar del Plata, and ANPCyT Grant No. PICT 0206/12 (Argentina).

${ }^{1}$ J. J. Binney, N. J. Dowrick, A. J. Fisher, and M. Newman, The Theory of Critical Phenomena: An Introduction to the Renormalization Group (Oxford University Press, Inc., New York, NY, USA, 1992).

${ }^{2}$ H. B. Callen, Thermodynamics and an Introduction to Thermostatics, 2nd ed. (Wiley, New York, NY, USA, 1985).

${ }^{3}$ K. Binder, Rep. Prog. Phys. 50, 783 (1987).

${ }^{4}$ W. Kauzmann, Chem. Rev. 43, 219 (1948).

${ }^{5}$ B. I. S. A. Z. Patashinskii, Sov. Phys. JETP 50, 712 (1979).

${ }^{6}$ B. I. S. A. Z. Patashinskii, Sov. Phys. Solid States 22, 655 (1980).

${ }^{7}$ S. B. Kiselev and J. F. Ely, Physica A 299, 357 (2001).

${ }^{8}$ A. Cavagna, I. Giardina, and T. S. Grigera, J. Chem. Phys. 118, 6974 (2003).

${ }^{9}$ E. S. Loscar, E. E. Ferrero, T. S. Grigera, and S. A. Cannas, J. Chem. Phys. 131, 024120 (2009).

${ }^{10}$ H. K. Janssen, B. Schaub, and B. Schmittmann, Z. Phys. B: Condens. Matter 73, 539 (1989)

${ }^{11}$ B. Zheng, Int. J. Mod. Phys. B 12, 1419 (1998).
${ }^{12}$ E. V. Albano, M. A. Bab, G. Baglietto, R. A. Borzi, T. S. Grigera, E. S. Loscar, D. E. Rodriguez, M. L. R. Puzzo, and G. P. Saracco, Rep. Prog. Phys. 74, 026501 (2011).

${ }^{13}$ E. S. Loscar, C. G. Ferrara, and T. S. Grigera, J. Chem. Phys. 144, 134501 (2016).

${ }^{14}$ A. Cavagna, Phys. Rep. 476, 51 (2009).

${ }^{15}$ L. Berthier and G. Biroli, Rev. Mod. Phys. 83, 587 (2011).

${ }^{16}$ P. H. Poole, F. Sciortino, U. Essmann, and H. E. Stanley, Nature 360, 324 (1992).

${ }^{17}$ O. Mishima and H. E. Stanley, Nature 396, 329 (1998).

${ }^{18}$ D. T. Limmer and D. Chandler, J. Chem. Phys. 135, 134503 (2011).

${ }^{19}$ P. Debenedetti, Metastable Liquids: Concepts and Principles (Princeton University Press, Princeton, NJ, 1996).

${ }^{20}$ S.-Y. Huang, X.-W. Zou, Z.-J. Tan, and Z.-Z. Jin, Mod. Phys. Lett. B 15, 369 (2001).

${ }^{21}$ S.-Y. Huang, X.-W. Zou, Z.-J. Tan, and Z.-Z. Jin, Phys. Lett. A 297, 105 (2002).

${ }^{22}$ P. J. Steinhardt, D. R. Nelson, and M. Ronchetti, Phys. Rev. B 28, 784 (1983).

${ }^{23}$ D. A. Huse, Phys. Rev. B 40, 304 (1989).

${ }^{24}$ P.-G. De Gennes and J. Prost, The Physics of Liquid Crystals (Oxford University Press, 1995).

${ }^{25}$ L. Fernández, J. Ruiz-Lorenzo, M. Lombardo, and A. Tarancón, Phys. Lett. B 277, 485 (1992).

${ }^{26}$ L. Schülke and B. Zheng, Phys. Rev. E 62, 7482 (2000).

${ }^{27}$ C. Anteneodo, E. E. Ferrero, and S. A. Cannas, J. Stat. Mech.: Theory Exp. 2010, P07026.

${ }^{28}$ C. Unger and W. Klein, Phys. Rev. B 29, 2698 (1984).

${ }^{29}$ W. Klein and F. Leyvraz, Phys. Rev. Lett. 57, 2845 (1986).

${ }^{30}$ H. Wang, H. Gould, and W. Klein, Phys. Rev. E 76, 031604 (2007).

${ }^{31}$ F. Trudu, D. Donadio, and M. Parrinello, Phys. Rev. Lett. 97, 105701 (2006).

${ }^{32}$ E. S. Loscar and E. V. Albano, Europhys. Lett. 85, 30004 (2009).

${ }^{33}$ J. R. Errington, P. G. Debenedetti, and S. Torquato, J. Chem. Phys. 118, 2256 (2003).

${ }^{34}$ J.-P. Hansen and L. Verlet, Phys. Rev. 184, 151 (1969).

${ }^{35}$ M. P. Allen and D. J. Tildesley, Computer Simulation of Liquids (Oxford University Press, 1989).

${ }^{36}$ L. A. Fernández, V. Martín-Mayor, B. Seoane, and P. Verrocchio, Phys. Rev. Lett. 108, 165701 (2012).

${ }^{37}$ S. Sarkar, R. Biswas, M. Santra, and B. Bagchi, Phys. Rev. E 88, 022104 (2013).

${ }^{38}$ P. R. ten Wolde, M. J. Ruiz-Montero, and D. Frenkel, Phys. Rev. Lett. 75, 2714 (1995).

${ }^{39}$ H. Tanaka, J. Stat. Mech.: Theory Exp. 2010, P12001.

${ }^{40}$ L.-Y. Chen, P.-H. Tang, and T.-M. Wu, J. Chem. Phys. 145, 024506 (2016).

${ }^{41}$ A. C. Y. Liu, R. F. Tabor, L. Bourgeois, M. D. de Jonge, S. T. Mudie, and T. C. Petersen, Phys. Rev. Lett. 116, 205501 (2016).

${ }^{42}$ D. Stauffer and A. Aharoni, Introduction to Percolation Theory (Taylor \& Francis, London Bristol, PA, 1994).

${ }^{43}$ V. K. Shen and J. R. Errington, J. Phys. Chem. B 108, 19595 (2004).

${ }^{44}$ P. Meakin, J. Colloid Interface Sci. 102, 491 (1984).

${ }^{45}$ D. Weitz and M. Oliveria, Phys. Rev. Lett. 52, 1433 (1984). 